\title{
An Extensive Study on Machine Learning based Battery Health Estimation
}

\author{
Sai Vasudeva Bhagavatula ${ }^{I}$, Venkata Rupesh Bharadwaj Yellamraju ${ }^{I}$, Karthik Chandra Eltem ${ }^{I}$, \\ Phaneendra Babu Bobba ${ }^{l}$ and Naveen Kumar Marati ${ }^{2}$ \\ ${ }^{1}$ Dept. of Electrical \& Electronics Engineering, GRIET, Hyderabad, Telangana., India. \\ ${ }^{2}$ Architect, Wipro Limited, Bangalore, India.
}

\begin{abstract}
This manuscript is a comparative study on various machine learning Regression methods like Decision Tree and Random Forest and SVM and other improvised methods along with unsupervised methods like Reinforcement learning, ANN methods like DNN are also discussed along with advanced methods like GRU, CNN, LSTM for estimating the battery health in order to estimate its life which is used in the modern-day technology of Battery Management System. The evolution of the present day BMS bought a great opportunity to study more about adaptive learning systems as it provides greater efficiency and tunes itself basing on environmental changes for battery health estimation studying on various methods on the subsets of artificial intelligence can be helpful to build more accurate correlation between the input and output. Adaptive learning even having a self-adjusting feature the computational limitations and the data being used is also important in producing correct result with a promising accuracy, so multiple algorithms, architectures and models are studied for better understanding in order to come to conclusions for selecting the apt model for satisfying results. Compared to other conventional methods Artificial Intelligence and their subsets learn from the error and adopt which outperforms other models in accuracy.
\end{abstract}

\section{Introduction}

A battery management system this helps to improve the robustness and performance of the battery. The BMS acquires information about the electrical and ambient parameters of the battery and not only protects it from electrical damages but also helps to enhances its functioning and battery life. The behavior of the battery can be understood by the State of Charge and State of Health or the Performance Characteristics are very useful is modern day technologies earlier many conventional methods were used for estimating these responses but later after the improvisation in the computational power of machines like computers old technologies like

Adaptive learning methods - machine learning and ANN (Artificial Neural Nets) started evolving that offer far better performance than the conventional approaches of estimating $\mathrm{SoC}$ and $\mathrm{SoH}$. A representation of various $\mathrm{ML}$ techniques and their classification are mentioned below.

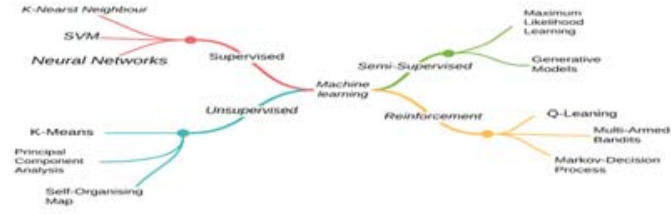

Fig 1. Representation of Machine Learning Methods

In the recent years the research in this particular field has grown enormous due its flexibility and accessibility.

From the vast research being conducted on these self- adjusting systems for the Battery health estimation some of basic attributes like voltage current and ambient temperature of the battery pack is being used for the estimation of SoC and for the estimation of health of the battery pack additional parameter like SoC, charge cycles, voltage, current, temperature and along with additional parameters are taken into consideration when dealing with these methodologies. A basic workflow any machine 
learning method is given below in Fig 2 in a block representation.

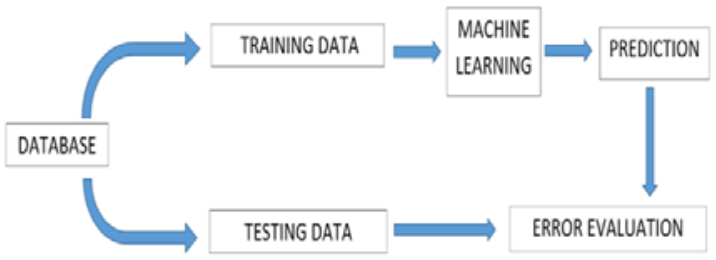

Fig 2. Block Representation Machine Learning process

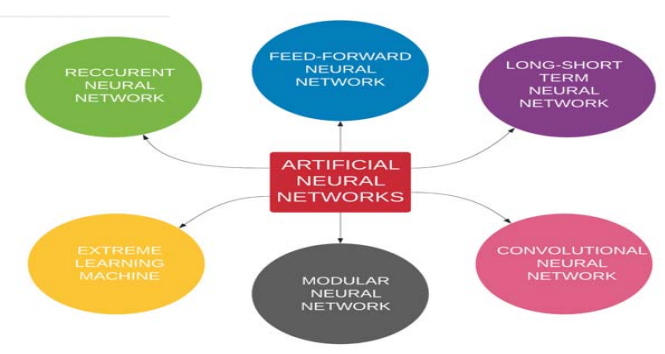

Fig 3. Types of ANN

Multiple ML and ANN frameworks along with their proposed methodologies are discussed below. This manuscript is extensive study on different methodologies in machine learning that are being experimented for the health estimation of the battery. A summary of the methods discussed in the below section is tabulated for better understanding

\section{Study on Different Machine Learning Approaches}

\subsection{Random Forest}

The Random Forest regression technique is an addition to the Decision [1]. The authors of [2] performed experiments on the data available.

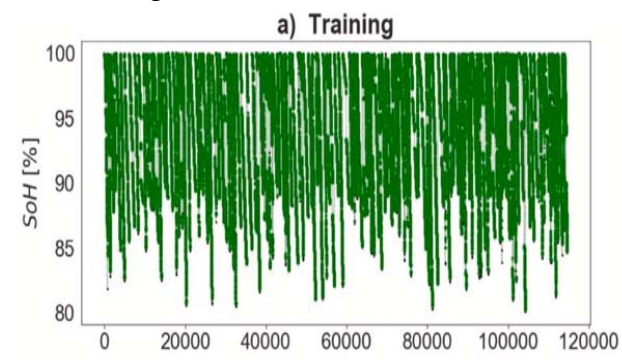

Fig. 4 (a). Training $\mathrm{SoH}$

Fig 4 (a) clearly represents the predicted SoH during training which is labelled in green colour, the original $\mathrm{SoH}$ is not visible as it is imposed with the true $\mathrm{SOH}$ which is label in black colour this concludes that the random forest regression model has been trained.

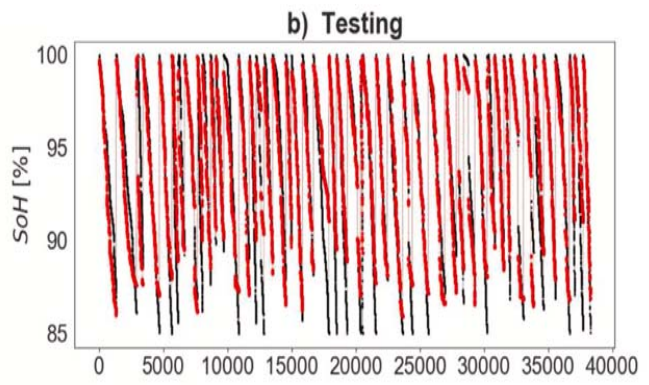

Fig 4. (b) Testing SoH

As observed in fig 4(b) the evaluation over the testing data is done and plotted The colour labelled in red represents the predicted data for the SoH over the validation data. As seen the black colour is the true SoH.

The results acquired were quite promising with a mean absolute error percentage of $1.27 \%$, using the Random Forest model selected by the authors of [2]. The authors of [3] describes how Decision Tress match the traditional Li-ion battery aging. More than 400 trees were selected but the authors of [2 ] for their random forest model. The error in the aging parameter of the battery can be understood by the decrease in mean loss in impurity significance during the computation. The authors of [4] describes about the significance of categorical values, This process is usually repudiated. The other parameter that has a strong influence and its durability on the accuracy of the model for aging estimation is the Permutation importance. [5] elucidates how the decrease in model accuracy is observed by the quantification parameter significance. In $[3,6,7]$ it is stated that loss of active components in the li-ion battery degrades the capacity of battery.

A similar type experiment was executed by the authors of [8] for the estimation of capacity on lithium ion power source banks like batteries using the random forest regression method their results states that the designated model with 200 trees were sufficient with the trained and tested data as listed by the authors of [8] in the table Setting 2 the estimation has output a very promising with a RMSE (Root Mean Square Error) percentage that is below $1.3 \%$. The observations fairly conclude the calibre of the RFR (Random Forest Regression ) model that it was able to understand the data provided in the form of training samples to the models.

The below are the results from NMC(nickelmanganese-cobalt ) batteries which are classified into two basing on their specifications and 6 groups of cells were chosen for each type. The below are the results from Type A specification cells. 


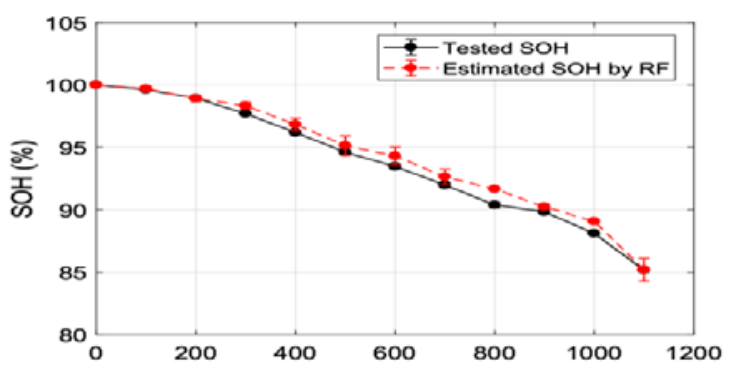

Fig. 5(a). Predicted Results for Group 1 Cells

The Fig 5(a) [8] are the graphs obtained for RFR for Group 1 cells the Predicted/ Estimated SoH vs the True or Tested SoH is plotted and we can observe that there is very less error that can be ignored when considering or trained on a huger dataset The $\mathrm{x}$ axis being its charging cycles and $\mathrm{Y}$ axis being its $\mathrm{SoH}$ the model was able to predict the State of Health even with less training data samples quite impressively

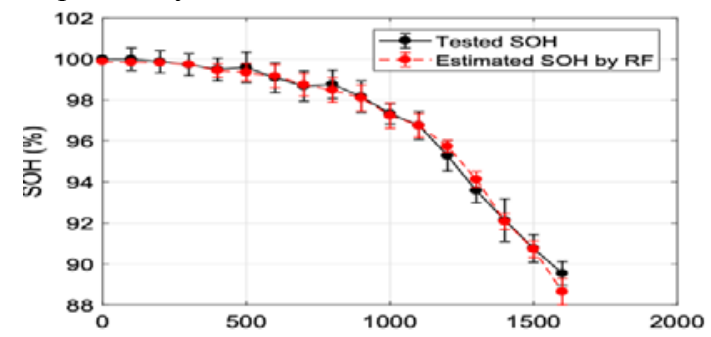

Fig 5 (b) Predicted Results for Group 4 Cells

The Fig 5(b) [8] for Group 4 cells the Predicted/ Estimated $\mathrm{SoH}$ vs the True or Tested $\mathrm{SoH}$ is plotted The $\mathrm{x}$ axis being its charging cycles and $\mathrm{Y}$ axis being its $\mathrm{SoH}$ the model was able to predict the State of Health even with less training data samples perfectly fit the true $\mathrm{SoH}$ which yields better results compared to Group 1 cells. more better prediction was made by the same model when evaluated with the Type B cells. The results below

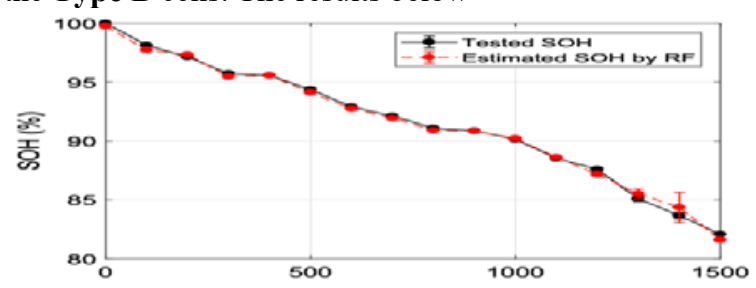

Fig 6(a) Predicted Results for Group 1 Cells

The SoH curves as observed in 6(a) were obtained from the Type B of the NMC cells the specifications are given by the authors of [8] as shown for the same model the SoH perfectly fits the true SoH state that concludes that are model is perfectly trained and was able to predict the $\mathrm{SoH}$

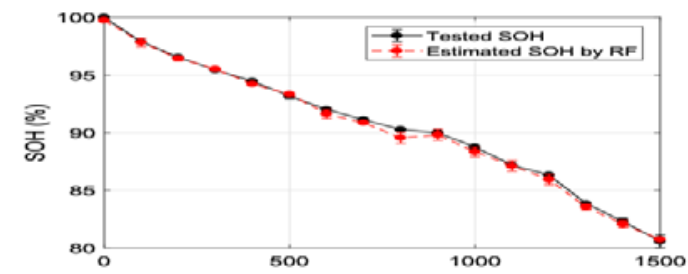

Fig 6(b) Predicted Results for Group 2 Cells

Same type of cells but of different groups were used by the authors of [8]. The $\mathrm{SoH}$ as per this curve in 6(b) comprehends that it was able to coincides with the original SoH. Making it clear that the model used by the author of [8] is the best fit for the $\mathrm{SoH}$ prediction for the selected NMC batteries with an RMSE error of less than $0.5 \%$ for type B batteries

\subsection{Support Vector Regression}

Many Artificial Neural Networks and Support Vector Regression illustrated as statistical and adaptive learning we used for the prediction/estimation have been successfully implemented for SoC [9-12]. The use of Support Vector Machine was already being discussed for the state of charge estimation by the authors of [13] for on board electric vehicles. Anticipating the life of the battery by SVM technique as shown by the authors of [14] was achieved, the proposed methodology was learning the decay of battery capacity for the necessary results. The authors cited in [15] has claimed to conduct an experiment on the battery specs as mentioned in Table 1 of [15]. The battery chosen was bought from [16].

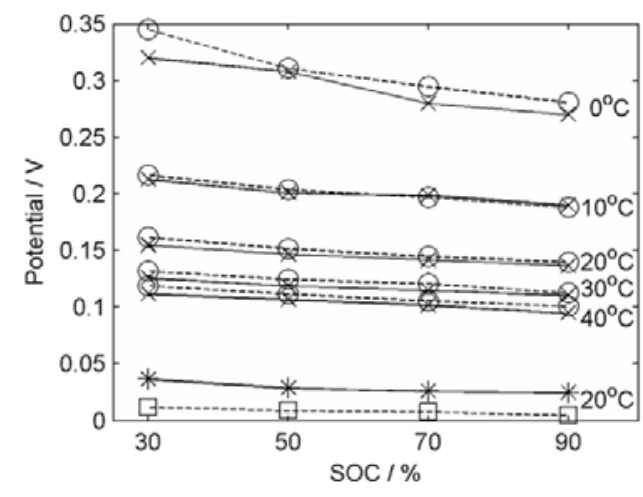

Fig 7(a) SoC vs Voltage

The authors of the reference [15] has plotted the curve for the voltage vs state of charge as shown in Fig 7(a) for different temperatures, the thick like is label for the true value of $\mathrm{SoC}$ and the dotted line is for the estimate value of SoC but the temperature has been neglected the test has been conducted for multiple temperatures varying from $0^{\circ} \mathrm{C}$ to $40^{\circ} \mathrm{C}$ with a step of $10^{\circ} \mathrm{C}$ and was used only for the true SoC neglecting the temperatures as a parameter for the SVM model the following were the results The curves contains additional parameters that were taken into consideration like instantaneous voltage and 
time dissociated voltage with a $\mathrm{x}$ and star for true and bubble and box for estimated respectively. A Root mean square percentage error of $4.2 \%$ and 0.85 for voltage ranging from 4.2 to 3 volts which is quite satisfying for time independent considerations.

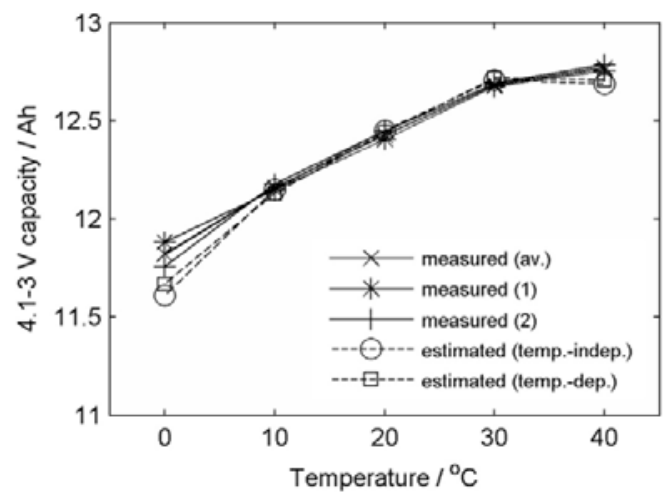

Fig 7 (b) Temperature Dependent SoC Estimation

The authors of [15] has also conducted a similar experiment for time dependent and achieved better results with a RMSE of $0.63 \%$ for voltage ranges and 6.2 at a particular instant voltage.

A similar experiment by the authors of [14] in their investigated for the $\mathrm{SoH}$ on lithium- ion batteries can be seen by considering the cells that were real time electrified transportation, 6 of these cells were taken for this real time experiment.

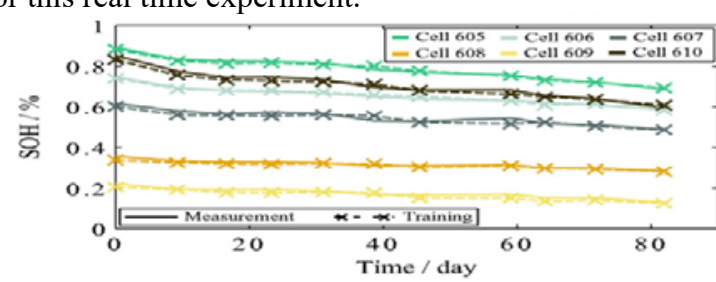

Fig 8(a) Training data $\mathrm{SoH}$ vs Time

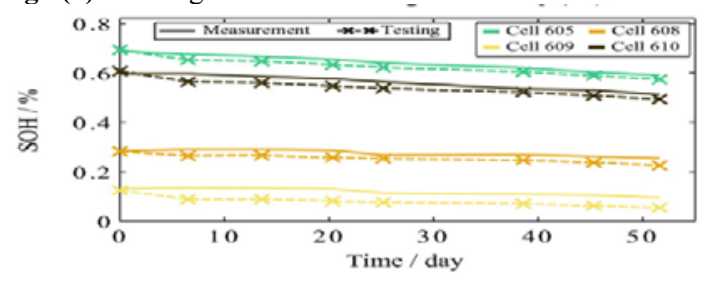

Fig 8(b) Testing data $\mathrm{SoH}$ vs Time

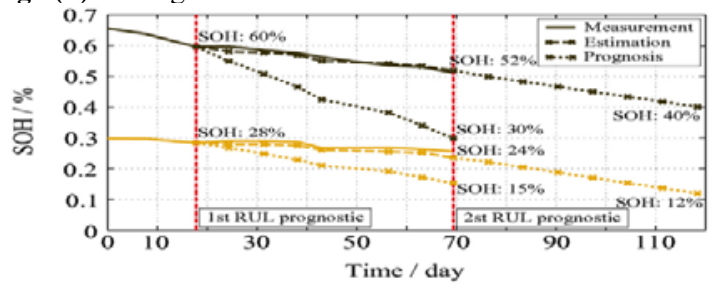

Fig 8(c) SoH vs Time Remaining Useful Life

The above were the real time results obtained for the authors of [14] Fig 8 [a] and Fig 8 [b] are the training and testing curves of the selected cell types, for more accurate validation authors of [14] have taken driving profiles HV1 to HV5 for training, HV5 driving profile was later removed when validating testing, For Remaining Useful Life estimation as seen in Fig $8(\mathrm{c})$ the process was conducted in a different manner, The RUL prediction started from the 12 cell prior to which training was conducted, and the $11^{\text {th }}$ cell was considered as reference for the first RUL computation and was continued for 70 days and using these data points of the first prognostic as shown in Fig 8(c) by the authors of 14 the data was then used for next 50 days of the second estimate as seen in the fig 8(c). A similar other experiment was done by the authors of [17], has collected datasets from the NASA data storehouse [18]. The authors [19] mentioned the training time for SVR.

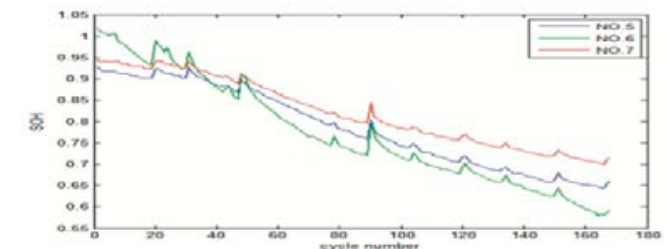

Fig 9 (a) $\mathrm{SOH}$ vs cycles

Fig 9(a) by the authors of [17] shows a capacity degradation curve for the TIECVD batteries as shown

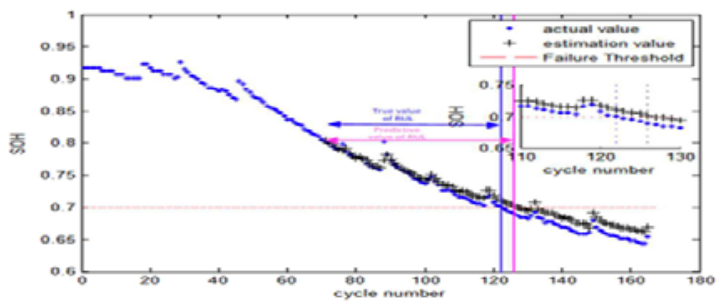

Fig 9(b) Predicted SoH vs cycles

The predict $\mathrm{SoH}$ curve as seen in the above figure 9(b) as done by the authors of [17] started their SVR model from cycle 70 we can observe that the SVR fits the true SoH affirmatively and approvable RMSE error of $1.28 \%$

which can be tuned and resolved by improvising the model.

\subsection{Deep Neural Networks}

In the previous section we have discussed about various machine learning algorithms. Authors of [20],[21] and [22] have proposed different ways for the State of Health Estimate. The authors of [23] in their proposed methodology has consider a classifier model for State of health estimate. Also, in [24] the State of Health from ranges $100 \%-75 \%$ it is experimented that for automotive industry the battery reaches will reach its expiry when it is below $80 \%$. The opted neural network classifier model [23] has 5 layers in total with 2 hidden layers of 10 nodes each, an input and an output layer. The inputs: voltage, temperature, state of charge, capacity and energy, and output nodes are 5 nodes classified from $75 \%$ to $100 \%$ with a $5 \%$ step each node for $\mathrm{SoH}$ indicators 
for example the one of the nodes of output layer ranges from $100 \%$ to $95 \%$ and another node $95 \%$ to $90 \%$ and so on.

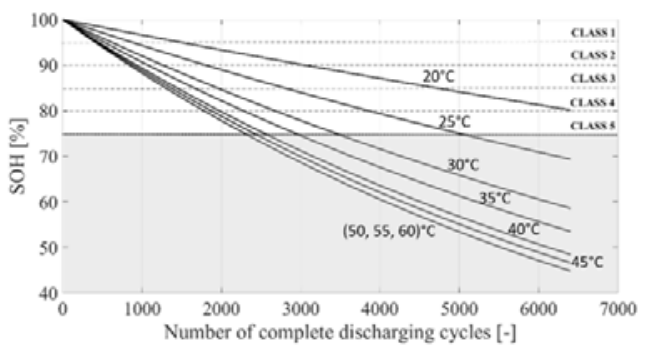

Fig 10 (a) SoH vs Cycle count

The fig 10 (a) as mention by the authors of [23] they have split the state of health into different classes or categories at different temperature ranges.

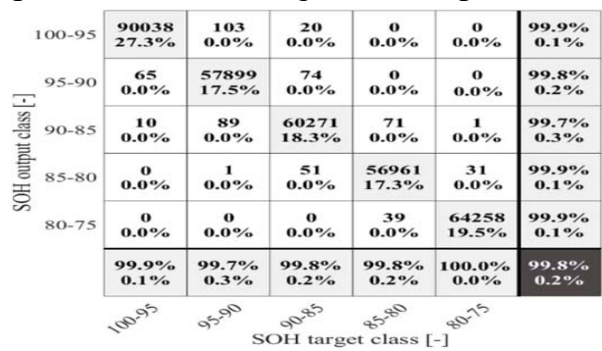

Fig 10 (b) Training

In the above fig 10 (b) by the authors of [23] which is a Training error matrix. The accurately classified State of Health are the diagonal elements, the wrongly classified State of Health are the elements other than the diagonal elements i.e., the off-diagonal elements.

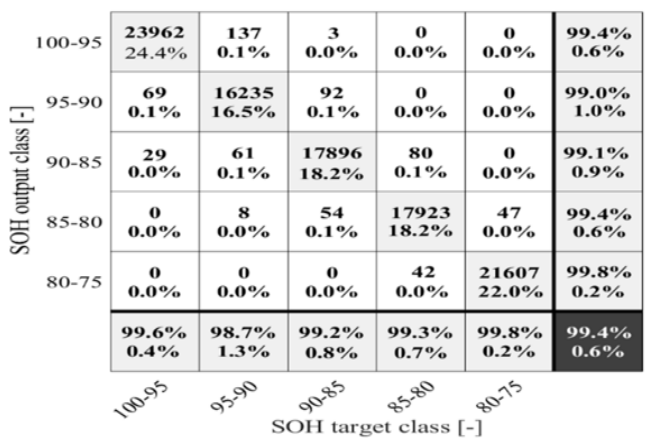

Fig 10(c) Validation

As seen in the above fig 10 (c) the matrix is a represented as an error matrix. The above are the validation results by the authors of [23]. The classification model has resulted in an accuracy of $99.4 \%$ for validated current profile 1 as taken by the authors of [23].

The authors of [21] has conducted an experiment for the State of health estimation for various batteries. The Neural Network model by the authors of [21] is a

3-layer back propagation model. The input layer in this model is given the arguments of the Equivalent Circuit Model of the battery [25],[26]. The neural network architectures were referenced by the authors of [22],[27],[28]. The SoH of below $80 \%$ has been mentioned in [29] by the authors is considered as an expiry mark.

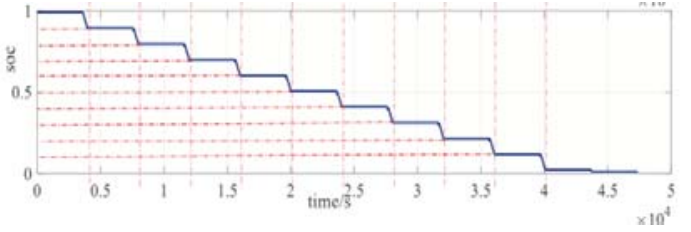

Fig $11 \mathrm{SoC}$ vs Time

Above figure 11 by the authors of 11 (a) is a $\mathrm{LiFePO}_{4}$ Battery and rated for $10 \mathrm{Ah}$ capacity. The authors investigated the results by considering a nominal temperature of $25^{\circ} \mathrm{C}$. The State of Charge vs Time as seen in the above can be comprehended that as usage time passes the SoC decays.

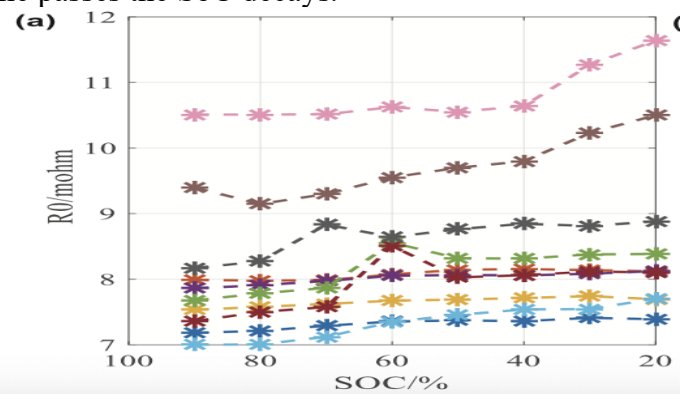

Fig 12(a) Ohmic Resistance vs SOC

From Fig 12(a) [21] there is an inversely proportional relation between the $\mathrm{SoC}$ and ohmic resistance.

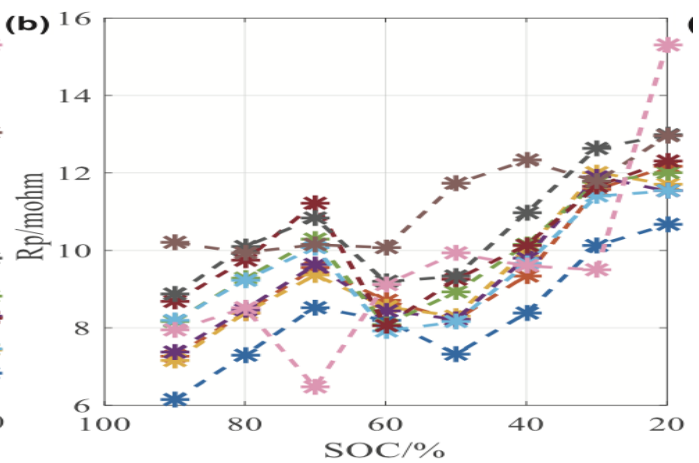

Fig 12(b) SoC vs Polarization Resistance

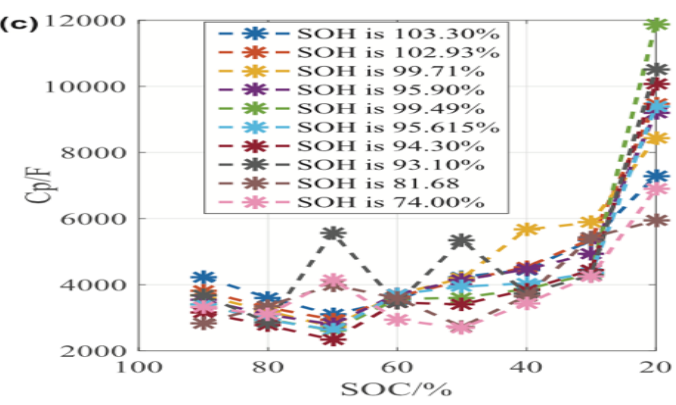

Fig 12(c) SoC vs Polarization Capacity

The figures 12(b) and 12(c) from [21] there is no direct relation so by applying a neural network algorithm we can find the relation between the SOC and both the Polarized Resistance and capacitance and further estimate the $\mathrm{SoH}$. The model used outputted an error of below $8 \%$.

\subsection{Gated Recurrent with} Convolutional Neural Networks 
The authors of [30] proposed a new multi architectural neural network for $\mathrm{SoH}$ forecasting. This new model is a blend of Gated Recurrent Unit which is GRU-RNN and Convolutional Neural Network which in short is CNN collectively in short is called GRU-CNN [31],[32]. This has the properties and functionalities of both CNN and GRU.

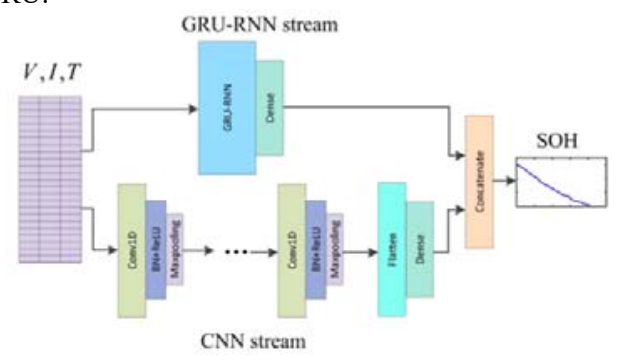

Fig 13 Schematic Architecture of GRU and CNN

A similar multi architecture like [31],[32],[35]. The datasets used by the authors of [30] were taken from [33] and [34]. Parameters like Voltage, Current and Temperature are considered at the input layer as shown in fig 13 ref [30].

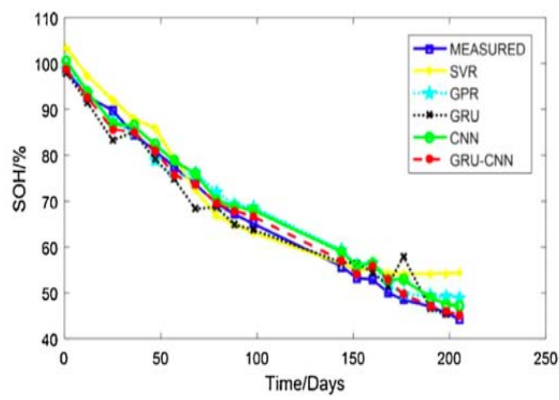

Fig 14(a) SoH vs Time NASA -RW 16

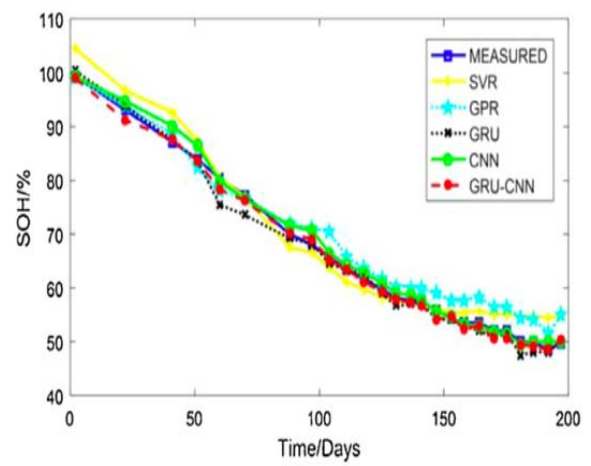

Fig 14(b) SoH vs Time NASA -RW 20

The SoH vs Days curve as shown above for the From Fig 14(a) and Fig 14(b) by the authors of [30] for NASA RW 16 and RW 20 batteries, we can also observe that other methods apart from GRU-CNN gave the results with the least error and highly fit the curve but proposed method fits the most with the true curve. The SoH Mean absolute error of $1.07 \%$ and $0.97 \%$ is achieved.

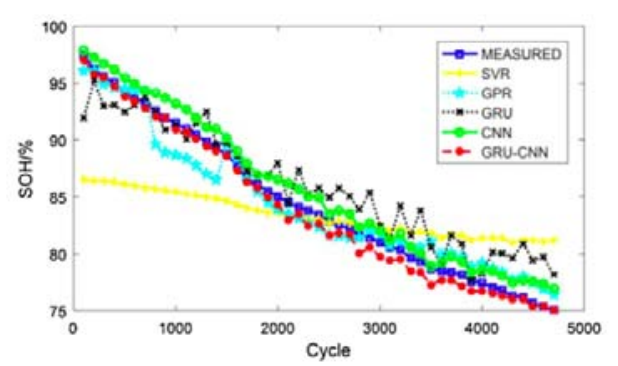

Fig 15(a) SoH vs Cycle OXFORD Cell 4

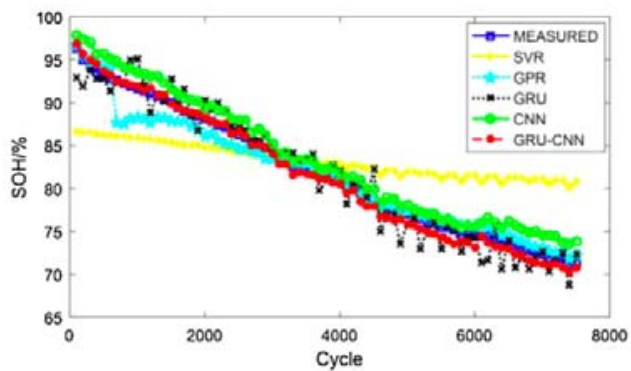

Fig 15(b) SoH vs Cycle- OXFORD Cell 8

Fig 15(a) and Fig15 (b) in [30] clearly shows the correlation of $\mathrm{SoH}$ and Cycles count for Oxford batteries

A very appreciating MAE of $0.61 \%$ and $0.65 \%$ has been achieved which is very less compare to other methods

We can understand that GRU-CNN yields better results in estimating $\mathrm{SoH}$ on the given parameters and performs more accurately for Oxford datasets.

\subsection{Convolutional Neural Networks with LSTM}

The proposed method by the authors of [36] is another kind of multi architectural neural network is a combination of both LSTM and CNN as discussed in the previous section where a similar such complex architecture is built using GRU and CNN. A similar such model for state of charge in reference [37-41] on datasets [33]. Below is a schematic representation of the model by the authors of [36].

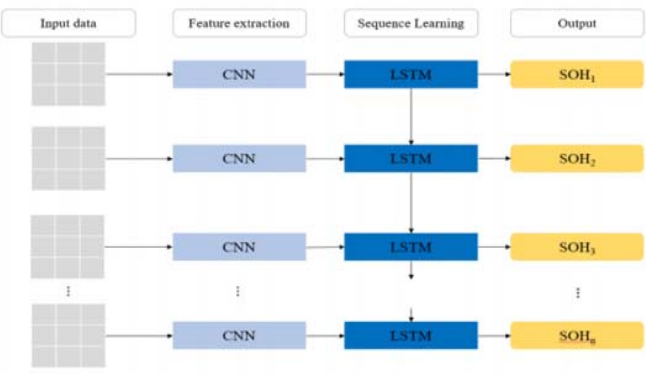

Fig 15 Schematic Architectural diagram of CNN-LSTM 


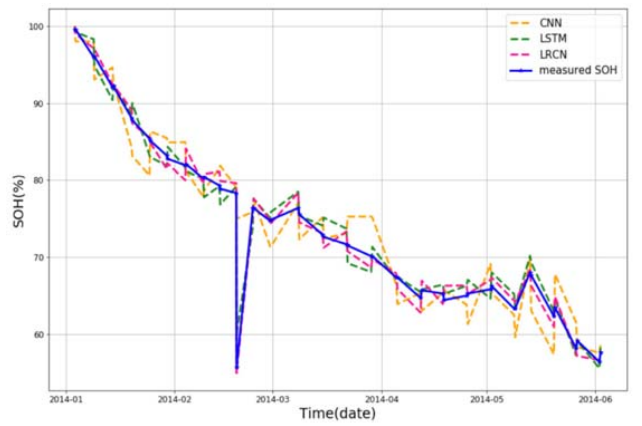

Fig 16(a) Soh vs Time

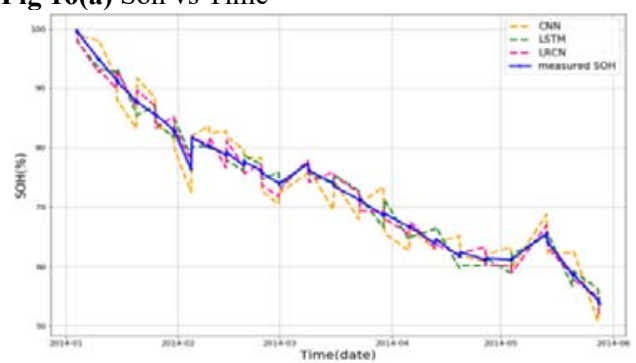

The figure 16(a) and 16(b) shows that the prediction by the Multi architecture proposed by the authors of [36] shows more accurate results than the other methods due to the saving capability of the model even there is loss of data it can predict the State of Health with a great accuracy classifying it to one of the best models for the estimation of $\mathrm{SoH}$ with an error of 1.09 RMSE and 1.51 MAPE respective which is very much reasonable.

Fig 16(b) SoH vs Time

Table 1 Summary Table

\begin{tabular}{|c|c|c|c|c|}
\hline $\begin{array}{l}\text { S.I } \\
\text { No. }\end{array}$ & $\begin{array}{c}\text { Machine Learning Model / ANN } \\
\text { Model }\end{array}$ & Description & Error & References \\
\hline 1 & Random Forest & 400 & $1.27 \%$ (MAPE) & [2] \\
\hline 2 & Random Forest & 200 & $\begin{array}{l}1.3 \% \text { (RMSE) } \\
<0.5 \% \text { (RMSE) }\end{array}$ & [8] \\
\hline 3 & $\begin{array}{c}\text { SVM } \\
\text { (Support Vector Machine) }\end{array}$ & $\begin{array}{c}\text { SoC Estimation } \\
\text { (Voltage Range } 4.2 \mathrm{~V} \text { to } \\
3 \mathrm{~V} \text { ) }\end{array}$ & $\begin{array}{c}0.85 \% \text { (RMSE) } \\
\text { and } \\
0.63 \%(\text { RMSE) }\end{array}$ & [15] \\
\hline 4 & $\begin{array}{c}\text { SVM } \\
\text { (Support Vector Machine) }\end{array}$ & Health Estimation & $1.28 \%$ (RMSE) & {$[14,17]$} \\
\hline 5 & $\begin{array}{c}\text { Deep Neural Networks } \\
\text { (Confusion matrix Classification) }\end{array}$ & $\begin{array}{c}5 \text { layers in total } 2 \\
\text { hidden layers of } 10 \\
\text { nodes each }\end{array}$ & $\begin{array}{l}0.6 \% \text { (absolute } \\
\quad \text { error) }\end{array}$ & [23] \\
\hline 6 & Deep Neural Networks & $\begin{array}{c}3 \text { layers } \\
1 \text { hidden layer with } 20 \\
\text { nodes each }\end{array}$ & $<5 \%$ (MPE) & [21] \\
\hline 7 & Gated Recurrent with CNN & $\begin{array}{l}\text { NASA RW } 16 \text { and RW } \\
20 \text { batteries }\end{array}$ & $\begin{array}{c}1.07 \% \text { (MAPE) } \\
\text { and } \\
0.97 \% \text { (MAPE) }\end{array}$ & [30] \\
\hline 8 & Gated Recurrent with CNN & $\begin{array}{l}\text { Oxford batteries } \\
\text { Cell } 4 \text { and Cell } 8\end{array}$ & $\begin{array}{c}0.61 \%(\mathrm{MAPE}) \\
\text { and } \\
0.65 \%(\mathrm{MAPE})\end{array}$ & {$[30]$} \\
\hline 9 & $\begin{array}{c}\text { Convolutional Neural Networks with } \\
\text { LSTM }\end{array}$ & Health Estimation & $\begin{array}{c}1.09 \text { (RMSE) } \\
\text { and } \\
1.51 \text { (MAPE) }\end{array}$ & [37] \\
\hline
\end{tabular}

\section{Conclusion}

The Anticipation of battery performance is a very important functionality in today's world as this helps the user to get acknowledged when to replace the battery. This paper is an extensive review of different advanced machine learning and ANN methods being used for the foretell of battery performance. BMS having its important functionality as computation of $\mathrm{SoC}$ and $\mathrm{SoH}$ can 
use such data driven methods as an evolutionary development. so, it can predict as per the data models. It is impossible to finalize a particular method for the need, as the there are many factors that affect the estimation like the data being used the computational power of the system, the selected model, the loss functions and many more all these play an equal role in finalizing the model it is impossible to tell that this model can be used. but overall, an error of around $1.5 \%$ is observed on all the methods. so with additional filtering and tuning we can reduce the error to some more extent and any of the methods can be used on the computational power of the processor.

\section{References}

1. Random Forests. Machine Learning 45, 5-32 (2001).

2. Kodjo S.R. Mawonou, Akram Eddahech, Didier Dumur, Dominique Beauvois, Emmanuel Godoy,

3. JPS, 484, (2021)

4. J. Vetter, P. Novák, M.R. Wagner, C. Veit, K.-C. Möller, J.O. Besenhard, M. Winter, M. WohlfahrtMehrens, C. Vogler, A. Hammouche, 147, 269(2005)

5. Strobl C, Boulesteix AL, Zeileis A, Hothorn T. Bias Bioinformatics. (2007) .

6. Strobl, C., Boulesteix, AL., Kneib, T. et al,. BMC Bioinformatics 9, 307 (2008).

7. Elie Riviere. Détermination in-situ,EE, (2016)

8. Samuel Pelletier, Ola Jabali, Gilbert Laporte, Marco Veneroni, 103,158 (2017),

9. Yi Li, Changfu Zou, Maitane Berecibar, Elise Nanini-Maury, Jonathan C.-W. Chan, Peter van den Bossche, Joeri Van Mierlo, Noshin Omar,232,197 (2018),

10. Terry Hansen, Chia-Jiu Wang,141, 351(2005),

11. J. C. Álvarez Antón, P. J. García Nieto, C. Blanco Viejo and J. A. Vilán Vilán, in IEEE Trans. on Pow. Elect., 28,5919, (2013)

12. Wu, Xuan, et al. AMRTTP, 21. (2011),

13. Wang Junping, Chen Quanshi, Cao Binggang,

14. ECM, 47, 858, (2006).

15. Verena Klass et al $2012 \mathrm{~J}$. Electrochem. Soc. 159 A 1856

16. Adnan Nuhic, Tarik Terzimehic, Thomas SoczkaGuth, Michael Buchholz, Klaus Dietmayer, JPS, 239,680, (2013),

17. Verena Klass, Mårten Behm, Göran Lindbergh, JPS, 270,262, (2014).

18. www.enerdel.com, April 16, (2013).

19. Qi Zhao, Xiaoli Qin, Hongbo Zhao, Wenquan Feng,MER, 85,99, (2018)

20. B. Saha, K. Goebel, , Moffett Field, CA, (2007).

21. Platt, J, MRT, Report (1998).

22. C. Chang, Z. Liu, Huang Y., Wei D. and Zhang L., CADDM, 24,22, (2014).

23. Duo Yang, Yujie Wang, Rui Pan, Ruiyang Chen, Zonghai Chen, EP, 105, 2059, (2017).

24. H. Lin, T. Liang and S. Chen, IEEE Trans. on Indus. Info.9,679, (2013)

25. Bonfitto, E. Ezemobi, N. Amati, S. Feraco, A. Tonoli and S. Hegde, (AEIT AUTOMOTIVE), 1, (2019).

26. S. Huang, K. Tseng, J. Liang, C. Chang and M. G. Pecht, Energies (2017).
27. He H, Xiong R, Guo H, et al. ECM, 64: 113, (2012).

Liu Z, He H, Applied Energy, (2015). Eddahech A, Briat $\mathrm{O}$, Bertrand $\mathrm{N}$, et al, IJEPES, 42(1),487, (2012).

28. Wang Y, Yang D, Zhang X, et al JPS, , 315: 199, (2016).

29. Dubarry M, Liaw B Y. Identify capacity fading mechanism in a commercial LiFePO 4 cell, Journal of Power Sources 194, (2009).

30. Yaxiang Fan, Fei Xiao, Chaoran Li, Guorun Yang, Xin Tang, JES, J,32, (2020).

31. F. Karim, S. Majumdar, H. Darabi, S. Chen, Lstm, IEEE Access, 1 (2017).

32. F. Karim, S. Majumdar, H. Darabi, S. Harford. 116,237, (2019).

33. B. Bole, C.S. Kulkarni, M. Daigle, Randomized battery usage data set, NASA Ames Progn. Data Repos. (2014).

34. R.B. Christoph, Diagnosis and Prognosis of, $\mathrm{Ph} . \mathrm{D}$. Thesis Department of Engineering Science, University of Oxford, (2017).

35. Z. Zhao, Y. Zheng, Z. Zhang, H. Wang, Y. Zhao, Proc. Interspeech,272 (2018),

36. S. Hong, M. Kang, H. Jeong and J. Baek, IECON The 46th Annual Conference of the IEEE Industrial Electronics Society,1929, (2020).

37. X. Song, F. Yang, D. Wang, K. Tsui IEEE Access, 7, 88894, (2019)

38. Kora, Padmavathi, and K. Sri Rama Krishna. "Bundle block detection using genetic neural network." In Information systems design and intelligent applications, pp. 309-317, (2016).

39. Vinay Kumar Awaar, Praveen Jugge and Tarakalyani S, Adv. in Elec. and Electronic Engg., 13,no.2, (2015).

40. V. K. Awaar, P. Jugge and Tara Kalyani S, (IEEE INDICON), New Delhi, (2015).

41. Sai Vasudeva Bhagavatula, Venkata Rupesh Bharadwaj Yellamraju, Karthik Chandra Eltem, Phaneendra Babu Bobba and Naveenkumar Maratii, E3S Web Conf., 184 (2020) 01068 